\title{
Megaminería, agua y territorio: procesos de des-re-territorial- ización frente al concesionamiento de zonas de importancia hídri- ca en la parroquia El Chical, provincia del Carchi, Ecuador
}

\section{Megaminery, water and territory: processes of un-re-territorial- ization due to concession of important water resources in zones of the parish of El Chical, Carchi province, Ecuador}

\author{
Mireya Levy ${ }^{1}$ \\ 凶 Autor para correspondencia: mireyalevy@hotmail.com \\ ${ }^{1}$ Universidad Central del Ecuador, Maestría en Gestión Integrada de Recursos Hidricos y Riego
}

\begin{abstract}
Resumen
Este artículo es un esfuerzo por definir y caracterizar las "zonas de importancia hídrica" afectadas por las concesiones mineras dentro del territorio establecido como área mestiza de la parroquia El Chical, en la provincia del Carchi. Frente a la ola de concesionamientos para minería industrial, surgen un sinnúmero de debates e incertidumbres por parte de las poblaciones locales que se encuentran en territorios definidos como mineros por el Estado central. Al estar las áreas concesionadas en conflicto con estas "zonas de importancia hídrica" se genera una competencia por los usos y manejo del territorio que, desde la perspectiva de esta investigación, implica una forma de desterritorializar a la población, por cuanto sus modos de vida y los recursos naturales para mantener los mismos se ven amenazados. Esta amenaza se materializa a través de la presencia, cada vez más visible, de empresas mineras trasnacionales, que van influyendo en las relaciones locales. Este artículo es la primera parte de una investigación más amplia, cuyo objetivo es analizar factores de desterritorialización y reterritorialización, desde los diversos actores, en relación con el recurso agua, sus usos y su conservación; como una respuesta a la imposición de territorios mineros, en la parroquia El Chical. En esta primera parte se realiza una caracterización de la problemática agua y minería; un análisis teórico de los procesos de des-reterritorialización desde el enfoque de la geografía crítica, para finalmente aterrizar con un análisis de la situación actual de concesiones y su solape con zonas de importancia hídrica en el área de estudio.
\end{abstract}

Palabras clave: Minería, Agua, Territorio, Des-re-territorialización

\begin{abstract}
This article is an effort to define and characterize "important hydrologic zones" affected by mining concessions in the territory defined as mestizo, part of the parish El Chical in Carchi province in northwestern Ecuador. Facing a wave of concessions for industrial mining, many debates and uncertainties from the local populations that inhabit these territories, now defined as miners from the central state, have emerged. As these new concessions enter in a conflict for land use, it generates deterritorialization of local habitants, since their way of living and the natural resources to maintain them are threatened by state politics. Besides this, the materialization of this politics, including the presence of transnational mining companies, have already influenced changes in
\end{abstract}


local relationships. This document is the first part of a wider investigation, whose objective is to analyze factors of deterritorialization and reterritorialization, from different actors perspectives, related to water, its use and conservation as a contestation to the imposition of mining territories, in El Chical parish. The document makes a characterization of the problematic between water and mining, and then analyzes from the critical geography perspective the processes of deterritorialization and reterritorialization, to finally make and overlap between mining concessions and important hydrologic zones in the study area.

Keywords: Minery, Water, Territory, Un-re-territorialization

\section{Introducción}

La historia de la megaminería en el Ecuador se inicia en la década de los 90 en el marco de la implantación de políticas neoliberales en Latinoamérica. En esta década empresas de prospección extranjeras adquieren millones de hectáreas para exploración geológica “... desde las orillas de la selva amazónica, en el sur del país, hasta los páramos andinos y los bosques húmedos de las vertientes occidentales de la cordillera de los Andes" (Sacher, 2017, p. 155). Desde este momento, los gobiernos que se sucedieron, tanto los del periodo neoliberal como el autodenominado gobierno de la "Revolución Ciudadana", promovieron el proyecto de transformar el Ecuador en un país de minería industrial. Durante las últimas dos décadas, los gobiernos ecuatorianos proveyeron un apoyo institucional, legal, político y moral a las empresas transnacionales.

Se diseñó entonces una "nueva institucionalidad minera" (Sacher, 2017) que permitió definir cinco proyectos megamineros metálicos prioritarios, calificados de "estratégicos"': Mirador, en Zamora Chinchipe, cuyo contrato de explotación se firmó en el 2012 con las transnacionales chinas Tongling y CRCC; Fruta del Norte, en Zamora Chinchipe, a cargo de la compañía sueco-canadiense Lundin Gold; Loma Larga, en la provincia del Azuay, a cargo de la canadiense INV Metals; Río Blanco, en la misma provincia, a cargo de la empresa china Junefields, y San Carlos-Panantza, en Morona Santiago, también a cargo de las estatales chinas Tongling y CRCC. Estos proyectos, según estimaciones del mismo gobierno, una vez que inicie su explotación, significarían exportaciones anuales por USD 3.778 millones, de los cuales el Estado recibiría USD 784 millones por

1 Estos proyectos se definen como estratégicos por el gobierno central, por ser los más avanzados, pero no son las únicas áreas donde se plantea la realización de actividades de minería industrial en el Ecuador (Sacher, 2012). concepto de regalías. Esto eventualmente llegaría a constituir el 5,6\% del PIB (Sacher, 2017, p. 172).

Además de estos proyectos, el gobierno procedió a la reapertura del Catastro Minero, en abril del 2016, lo que dio paso a una nueva ola de concesionamientos para minería a gran escala a lo largo y ancho del país, que abarca aproximadamente un $15 \%$ del territorio nacional (ARCOM, 2018). Estas nuevas concesiones a nivel nacional, causaron descontento en las comunidades cuyos territorios se encuentran afectados, especialmente debido a la falta de información, y la inexistencia de consultas previas. Dentro de este $15 \%$, se incluye el concesionamiento de más del 30\% de áreas definidas como Bosques Protectores, categoría de protección bajo la cual la realización de actividades extractivas no está prohibida (Vandergrift et al., 2017).

El noroccidente de la provincia del Carchi, es uno de los "bloques" definidos por el Ministerio de Minería (Ministerio de Minería del Ecuador, 2016). Dentro de este bloque la parroquia El Chical, específicamente, tiene más de un $90 \%$ de su territorio parroquial concesionado, para lo que según el catastro minero se define como actividades de "mediana y gran minería" (Ministerio del Ambiente Ecuador, 2017). Esta zona, constituye uno de los últimos remanentes de bosque nativo de las estribaciones de la cordillera occidental, que forma parte de la región del Chocó (Gobierno Provincial del Carchi, 2015), pero además es una zona agrícola y pecuaria con unidades productivas familiares que dan sustento a más de 5.000 habitantes (GAD Parroquial El Chical, 2015). Así, los objetivos de la presente investigación son: por un lado, analizar los impactos de la minería en relación al recurso agua, en cuanto a su acceso, calidad y los cambios en su gobernanza. Estos impactos se abordan con los conceptos de des-reterritorialización, desde el enfoque de la geografía crítica, y se ha logrado aterrizarlos en el territorio en cuestión, al identificar zonas 
de importancia hídrica² y cómo estas se encuentran en un claro conflicto por el uso del territorio, al estar solapadas con concesiones mineras.

\section{Megaminería y sus impactos en el elemento agua}

El agua, dentro de las actividades mineras, es un punto álgido de conflicto con las poblaciones directamente afectadas por estas actividades, no solamente por el uso intensivo y de grandes cantidades del líquido vital que esta industria requiere, sino también debido a que las actividades mineras alteran la calidad y cantidad de agua disponible (Sacher \& Báez, 2011). Además, esta actividad genera cambios y transformaciones irreversibles en las formas institucionales y locales de regular y gobernar este elemento (Budds, 2012; Sosa \& Zwarteveen, 2014).

Una parte del agua es requerida por la actividad minera para el lavado y triturado de rocas que contiene el mineral a extraerse, pero a su vez, el drenaje acido de mina y los diques con agua contaminada son un riesgo constante de contaminación a gran escala ${ }^{3}$, así como la extracción de agua subterránea que altera flujos de vertientes aguas abajo, deviniendo en profundas modificaciones en los regímenes hídricos (Sosa \& Zwarteveen, 2014; Sacher \& Acosta, 2012; Yacoub, 2007).

Pese a la existencia tanto de normativas y regulaciones nacionales como tratados internacionales, en referencia a la gestión del agua en operaciones mineras, la alteración drástica de los recursos hídricos, flujos y ecosistemas acuáticos, según la experiencia de países como Perú es, en palabras de Bebbington \& Williams (2008), "incuestionable". Donde se implementa la minería industrial, aumenta la conflictividad social y socio-ambiental en relación con esta industria (Sairinen, Tiainen \& Mononen, 2017). Esta situación se agrava tomando en cuenta que las concesiones mineras a menudo abarcan zo-

2 Zonas de importancia hídrica: áreas con alto potencial para recarga, zonas de conservación bajo diferentes categorías cuyo mantenimiento es importante para regular y mantener los recursos hídricos, fuentes donde existen captaciones para diferentes usos del agua.

3 Entre los mayores desastres por colapso de diques de colas se encuentran: Summitville Gold mine, en EE.UU., en el año 1986; Mina de Oro Omai, en Guyana, en el año 1995; Mina Ashanti Compañía Goldfields, en Ghana, en el año 1996; Mina Gold Quarry, en EE.UU., en el año 1997; Mina Aurul, en Romania, en el año 2000; Mina Samarco, en Brasil, en el año 2015 (Burritt \& Christ, 2018). nas de cabeceras de cuenca, generando impactos a territorios ubicados aguas abajo (Yacoub, 2013).

"A través del agua, los efectos de la minería son experimentados en localidades que son distantes de la mina(s) y que de otra manera no serían afectados por la minería" (Budds, 2012, p. 128).

En cuanto a la evaluación de los impactos de la actividad minera sobre los recursos hídricos, la credibilidad sobre mediciones y datos de la cantidad y calidad del agua afectada por esta actividad, normalmente es realizada por actores externos al territorio, contratados por la empresa minera o, a su vez, son realizados por ella misma. Esto le confiere autoridad a la empresa para decidir y gestionar el agua sobre el territorio en el que se encuentra y evaluar los impactos de su propia actividad (Sosa \& Zwarteveen, 2012; Budds, 2012). Como consecuencia, denuncias sobre afectaciones al agua no se investigan ni sancionan, pese a que la población local maneja conocimientos de mucha más larga data, basados en su relación directa, siendo estos menospreciados como datos empíricos (Sosa \& Zwarteveen, 2014).

\section{Megaminería y procesos de des- reterritorialización}

\subsection{Fundamentos teóricos}

La conducción teórica de esta investigación se apoya en los conceptos que desde la geografía crítica se plantean para definir territorio y territorialidad. Estas definiciones son necesarias para analizar los procesos de des-re-territorialización, cuya comprensión se da a partir de estos dos conceptos previos. A través de este enfoque se busca comprender las dinámicas territoriales desde los diferentes actores y cómo se generan las diferentes territorialidades. La existencia de múltiples territorialidades implica una disputa por defender su legitimidad sobre la base de diferentes herramientas y estrategias.

Dentro de las ramas de la geografía, la geografía crítica parte de la comprensión del espacio habitado, no solamente como la base material para la supervivencia humana. Le confiere la categoría de "campo político en disputa" (Murillo \& Sacher, 2017); lo que implica que tanto el territorio como la naturaleza son categorías producidas socialmente desde el ámbito material y simbólico. Esta producción responde a diferentes intereses y lecturas del espacio natural y social que buscan apropiarse de éste. Esta aproxima- 
ción al espacio introduce una “(...) reflexión sobre cómo lo pensamos, lo representamos, lo vivimos y lo creamos" (Casellas, 2010, p. 575).

El concepto de territorio en América Latina ha sido utilizado de manera tanto descriptiva como analítica, por múltiples actores sociales, activistas y académicos (Sandoval, Robertsdotter \& Paredes, 2017). Estos actores van desde el Estado en su afán de territorializarse a través de las políticas públicas; las empresas transnacionales intentando controlar territorialmente mercados y recursos naturales; como los movimientos socio-ambientales y actores locales en respuesta a la confrontación entre estas fuerzas divergentes y de intereses contrapuestos (Fernandes, 2008).

El territorio se conceptualiza como un espacio apropiado, espacio hecho cosa propia, instituido por sujetos y grupos sociales que se afirman por medio de él (Porto-Gonçalves, 2009, p. 127), reconociendo las relaciones de poder inscritas en el espacio que se traducen en prácticas de control y apropiación (Sandoval et al., 2017). El territorio, como afirma Haesbaert (2007), es material y simbólico, y es a través de acciones simbólicas e instrumentales de apropiación y valoración que se produce la territorialización de un espacio (Raffestin, 1980). El territorio está constituido por diferentes territorialidades que se definen como el impulso e intencionalidad de los diversos grupos de imprimirse en el espacio (Haesbaert, 2013). Por lo que la territorialidad es construida entre la gente y el espacio natural, a través de acciones, percepciones, formas de valoración territorial y actitudes (Raffestin, 1980). El concepto de territorialidad se puede entender como una relación más dinámica, que implica prácticas, símbolos y manifestaciones que los grupos sociales, actores estatales, empresas, entre otros, realizan para definir sus territorios (Murillo \& Sacher, 2017).

Al hablar de la creación de territorios mineros, estos son configurados a través de las acciones emprendidas por el Estado desde los discursos, la propaganda, la institucionalidad. Pero además se hace referencia a políticas concretas que influyen en la infraestructura, los servicios, la determinación de categorías de ordenamiento territorial y vocación de usos del suelo para materializar su imaginario de Ecuador como un país de minería industrial; como a su vez la conversión de este sector en un importante rubro en la economía nacional.

Estos nuevos territorios mineros, definidos desde el ordenamiento territorial estatal (Ministerio de Minería del Ecuador, 2016), dan cuenta de una comprensión del territorio como un espacio donde lo legítimo se define solo desde el Estado central, a través de la gobernabilidad. Esto deviene en que las diversas territorialidades existentes dentro del territorio nacional se mantienen subordinadas, resultando en una relación de territorios dominantes y dominados “ $(. .$.$) eliminando los sujetos y las relaciones$ sociales que no son incorporados o captados dentro de los intereses del Estado" (Fernandes, 2008, p. 12).

\subsection{Des-reterritorialización}

A partir de la definición de territorio desarrollada, se parte de la premisa de que en un mismo espacio geográfico material confluyen diversas territorialidades. La convivencia o confrontación de estas territorialidades deviene en una producción constante de desterritorializaciones y reterritorializaciones sucesivas. Al ser las territorialidades dinámicas en sí mismas, estos procesos de des-re-territorialización no son situaciones estáticas sino procesos antagónicos en constante contestación.

Así, des-re-territorialización es una forma de enmarcar, imaginar, definir y clasificar territorios en los que se presentan actividades en competencia, lo que va a causar conflictos entre dos o más grupos, y donde uno de los grupos siente que la propuesta de su oponente es destructiva (el grupo desterritorializado), mientras el otro se legitima e impone (Holifield $\&$ Day, 2017). Esto llevado a un caso más específico como la territorialidad que representa la megaminería, implica que cuando esta actividad ingresa en un determinado territorio, resulta en una disputa que se define por el "carácter antagónico de las territorialidades y territorializaciones comunitarias, por una parte, y las de las grandes empresas mineras y el Estado, por otra" (Murillo \& Sacher, 2017, p. 52). Este proceso está marcado por diversos mecanismos que el Estado y las empresas mineras utilizan, a través de los cuales se pretende anular las territorialidades locales o llegar a un estado de adoctrinamiento de éstas, tomando en cuenta que las mismas no se extinguen del espacio. Esto quiere decir que ““”(...) los procesos de desterritorialización y reconstrucción de territorios, se encuentran en una dialéctica permanente entre ambos" (Haesbaert, 2013, p. 10).

Tal como el mismo Haesbaert lo analiza, existen múltiples formas de leer e interpretar la desterritorialización, sobre todo si el análisis se sitúa en la época actual de fase de sobreacumulación del capitalismo y consiguiente expansión de las actividades extractivas 
a nivel global (Sacher, 2017). Es, entonces, desde sus inicios, el capitalismo como sistema económico, un sistema que desterritorializa los modos de producción preexistentes para reterritorializarse.

Mientras que lo que se define como reterritorialización aparece como una contestación constante, más allá de la actividad de megaminería; es decir, es histórica y propia de la respuesta de la territorialidad que está siendo repelida o anulada. Esta emerge desde las territorialidades de las comunidades y pobladores que se presentan territorializados en áreas que han sido "(...) marginalizadas por los diferentes periodos de reproducción capitalista y que, justamente por eso, pudieron ser ocupadas por los pueblos racializados tras los sucesivos procesos de des-re-territorialización resultantes de los diferentes frentes de expansión" (Silveira, 2011, citado en Silveira et al., 2017, p. 71); o por el capital, en su afán de ocupar otros espacios.

La reterritorialización, entonces, es un movimiento de resistencia o respuesta a la desterritorialización que busca mecanismos de legitimar las territorialidades existentes, cuando se habla de que grupos previamente establecidos en un territorio son amenazados con ser segregados, o efectivamente pierden su capacidad de construir y ejercer control efectivo sobre sus territorios. Pero, además, las mismas actividades extractivistas, así como el Estado, pueden también re-territorializarse buscando re-ensamblarse, re-contextualizarse y re-crearse (Colectivo de Geografía Crítica Ecuador, 2017).

\section{Concesionamiento para megaminería de zonas de importancia hídrica4 en la parroquia El Chical, provincia del Carchi}

\subsection{Metodología}

Para determinar las "zonas de importancia hídrica", dentro del área de estudio definida, se identificaron usos del agua, áreas en conservación y zonas con alto potencial para recarga hídrica. Para identificar estas últimas se utilizó la metodología planteada por Matus, Faustino y Jiménes denominada "Guía participativa para la determinación de zonas de recarga hídrica" (Matus, Faustino, \& Jiménes, 2009) con base en información secundaria de parámetros físicos: pendiente, tipos de suelos, cobertura permanente y tipos de rocas que definen áreas potenciales para la recarga hídrica. Los resultados de la aplicación de esta metodología, luego se evalúan con los pobladores locales.

La realización de trabajo de campo consistió en identificar tanto zonas de reserva para cuidado de los recursos hídricos, como fuentes hídricas que están bajo alguno de los usos determinados ${ }^{5}$. Esto implicó el mapeo y visita a las captaciones de agua para consumo humano en los cinco centros poblados mestizos identificados dentro del área de estudio, y la identificación de áreas determinadas por las Juntas de Agua de Consumo como zonas de conservación de sus fuentes. Además, se realizaron cuatro talleres de mapeo participativo donde se identificaron, en el ámbito de la comunidad, las áreas prioritarias para manutención y uso de los recursos hídricos. Por último, se efectuaron entrevistas semiestructuradas a actores locales, en las cuales se dialogó sobre el manejo del agua dentro de cada comunidad, el conocimiento sobre las concesiones mineras y cómo estas influyen en las dinámicas del territorio y se asistió a tres asambleas, dos socializaciones de la empresa Cornerstone $^{6}$ y una marcha llevada a cabo en la ciudad de Tulcán.

Para conocer cuáles eran estos actores comunitarios se realizó la identificación de informantes claves con los que se iniciaron las entrevistas semiestructuradas. Estos informantes en un inicio fueron los presidentes/as de las cinco comunidades dentro del área de estudio (Unthal, Puerramal, El Chical, Quinshull y La Esperanza) y los presidentes/as de las Juntas Administradoras de Agua Potable de estas mismas comunidades, a quienes se entrevistó según su disponibilidad de tiempo. Además, a través de la asistencia a asambleas comunitarias relacionadas a la temática minera, se grabó intervenciones de otros actores que también forman parte de este proceso de mapeo. Las intervenciones fueron tanto de actores comunitarios, funcionarios
4 Zonas potenciales de recarga: áreas con la capacidad de almacenar agua en la superficie, que son conservadas y manejadas a fin de mantener sus características físicas de permeabilidad e infiltración, ya que afectan la magnitud de la recarga, y genera contaminantes que se puedan infiltrar al acuífero y dañar la calidad de sus aguas (Matus, Faustino, \& Jiménes, 2009). nía alimentaria.

6 Esta empresa canadiense es la operadora del Proyecto Espejo, compuesto por las concesiones Espejo 1, 2, 3 y 4. El titular de estas concesiones es la Empresa Nacional Minera (ENAMI). 
públicos, así como de trabajadores de empresas mineras presentes en el territorio.

Para completar el mapeo, los actores a nivel de instituciones públicas y empresas privadas, fueron identificados primero a través de las carteras de Estado encargadas de la política pública relacionada a la minería, agua y ambiente en el área de estudio: Gobierno Provincial del Carchi, GAD Parroquial El Chical, Ministerio de Minería (hoy Subsecretaría de Minería), Ministerio del Ambiente, Secretaría Nacional del Agua, Cámara de Minería del Ecuador, Empresa Cornerstone. De las grabaciones realizadas en asambleas y en las intervenciones, una vez transcritas se analizó qué instituciones no habían intervenido en estos espacios, por lo que era necesario solicitar entrevistas.

\subsection{Resultados}

Un análisis, a partir del catastro minero disponible en el sitio web de la Agencia de Regulación y Control Minero (ARCOM), permite visualizar que para la parroquia El Chical, las concesiones otorgadas y en trámite cubren áreas de cabeceras de cuencas hidrográficas, zonas de recarga hídrica, bosques protectores, zonas de producción agrícola y centros poblados donde las "territorialidades" existentes entrarían en posible contradicción con las actividades mineras que se quiere emprender allí.

Como se observa en la Figura 1, más del $90 \%$ del territorio parroquial se encuentra concesionado para minería a mediana y gran escala, a la Empresa Nacional ENAMI, a CARNEGIE RIDGE RESOURCES S.A, SEMIPROHG S.A. y COMPANÍA ECCOLMETALS S.A.

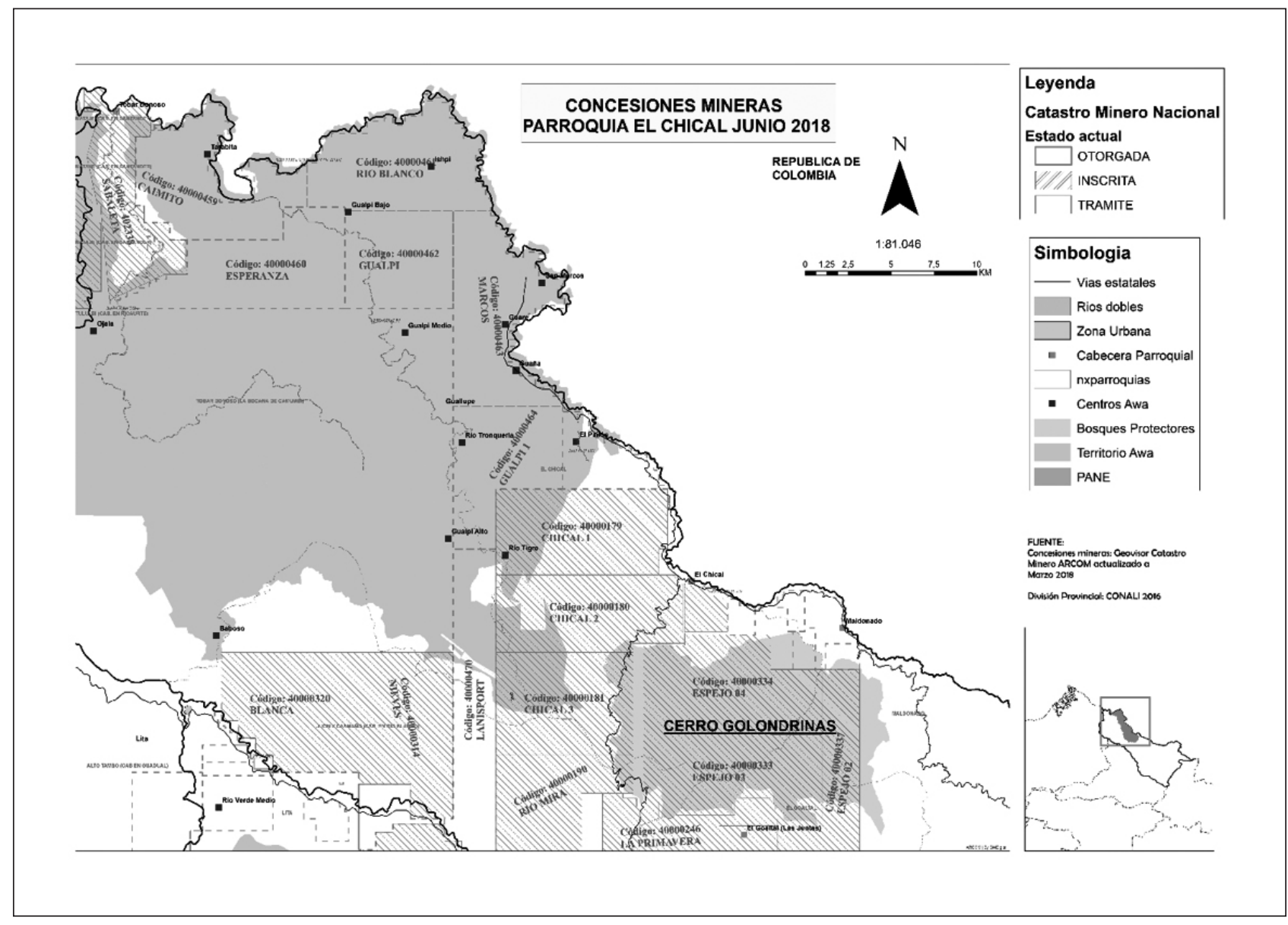

Figura 1. Concesiones mineras en la parroquia El Chical. Elaboración propia a partir de: Información Base, catastro minero ARCOM, IGM, MAE.

7 En las entrevistas realizadas a los dirigentes locales, se menciona que la zona nunca ha sido minera, ellos se definen como campesinos. Sobre los efectos de la minería saben por experiencias de las zonas de Tobar Donoso y Esmeraldas, que la contaminación de las fuentes hídricas es un grave problema, tanto como las problemáticas sociales. 
Dentro de esta zona, se definieron las siguientes áreas de conservación: el Bosque Protector Cerro Golondrinas, declarado dentro de esta categoría por el Ministerio del Medio Ambiente en 1995, con una extensión total de 13.509 ha (Gobierno Provincial del Carchi, 2015). Este es cabecera de ocho subcuencas hidrográficas que proveen agua para consumo humano, actividades productivas y equilibrio ecológico de manera directa a cuatro parroquias del noroccidente del Carchi (Gobierno Provincial del Carchi, 2015). El área conocida como Cerro Colorado, que constituye un espacio de conservación comunitaria de la comunidad La Esperanza. A su vez dentro del área se encuentran cinco Juntas Administradoras de Agua Potable de las cuales una mantiene una reserva hídrica de 5,29 y existen 705,5 ha en conservación dentro del programa Socio Bosque (MAE, 2017)

A través del cruce de los parámetros planteados por Matus et al., se determinan las zonas con mayor importancia para recarga de los sistemas hidrológicos de la zona. Las áreas con más alto potencial se encuentran hacia la zona sur de la parroquia y abarcan las áreas correspondientes al bosque protector Golondrinas, y a la cordillera conocida como Cerro Colorado más hacia el noroccidente (Figura 2). El factor uso del suelo, permite ubicar a estas como zonas prioritarias, por cuanto aquí están las áreas de remanente de bosque más conservadas a nivel parroquial. Coincidente además con las zonas de cordillera donde nacen las microcuencas que forman parte de la subcuenca binacional del río San Juan, mismo que es el límite internacional entre Ecuador y Colombia.

Según la definición de zonas de importancia hídrica contemplada para esta investigación, estos resultados se contrarrestaron con talleres de mapeo participativo, en los cuales la población identificó, dentro de los límites de sus comunidades, cuáles son las áreas más importantes para la conservación de los recursos hídricos. Las cinco comunidades visitadas concuerdan en que el área donde nacen las vertientes y donde se realiza la captación de agua para consumo humano es vital para la manutención de la calidad de vida en la zona. Además de esto se identifica que en las fincas se utiliza el agua para actividades piscícolas y abrevaderos, actividades no registradas por la SENAGUA al no contar con permisos de uso y aprovechamiento. Por último, tanto en el ámbito de talleres como de entrevistas personales se identifica al Bosque Protector Golondrinas como un área vital para la manutención de los sistemas hídricos en el área.
"El cerro Golondrinas para nosotros la nacionalidad Awá, el cual lo hemos mirado como nuestra única madre protector de esta zona del Carchi" (poblador Awá 2, grabación realizada por la autora, 9 de agosto 2017).

"Peormente vamos a permitir nuestras áreas que estamos protegiendo como es el cerro Golondrinas a donde nosotros somos agricultores, y tenemos nuestras aguas cristalinas que las tomamos en las dos parroquias como es Chical y Maldonado" (poblador comuna La Esperanza 3, grabación realizada por la autora, 16 de febrero 2018).

"No hemos sufrido del agua, tenemos bastante agua en toda la parroquia [El Chical]. Venimos cuidando el Bosque Protector Golondrinas, que no entre la gente minera. Nosotros siempre hemos manejado el agua, limpia, sana" (poblador Puerramal 1, grabación realizada por autora, 23 de marzo 2018).

Una de las razones más fuertes para definir esta apropiación se da porque allí se encuentran las 'nacientes' de las micro cuencas que alimentan las zonas pobladas en la región.

La categoría de bosque de vegetación protectora implica que no es prohibida la realización de actividades extractivas, según la legislación ambiental nacional. El Código Orgánico Ambiental, en su art. 89 define que los bosques y vegetación protectores se encuentran dentro del Patrimonio Forestal Nacional. Al estar en este subsistema de protección, la prohibición de realizar actividades extractivas de recursos no renovables no se aplica. Así, el artículo 54 del mismo cuerpo legal dictamina que solamente las áreas dentro del Sistema Nacional de Áreas Protegidas y en zonas declaradas como intangibles tienen prohibición de estas actividades. Por lo tanto, pese a que los bosques y vegetación protectores estén conservando zonas altamente frágiles, degradadas y que generan servicios ambientales vitales para poblaciones humanas, estos no tienen una protección en el ámbito de las leyes.

Otra categoría identificada como conservación corresponde a las áreas de conservación comunitaria y local, como el Bosque Cerro Colorado y el área en conservación perteneciente a la Junta de Agua Potable de El Chical. Estas fueron delimitadas por los pobladores interesados en su conservación. La primera se caracteriza por ser una zona de fuertes pendientes que dificulta su utilización para agricultura, pero además donde se ubican las 'nacientes' de tres micro cuencas. 
Este bosque comunal está escriturado a nombre de la comunidad La Esperanza y es un espacio territorial defendido por su importancia para mantener servicios ambientales. Sin embargo, el 100\% de su extensión se encuentra dentro de concesiones mineras; específicamente las concesiones Chical 1 y Chical 2, otorgadas a la empresa Carnegie Ridge Resources S.A.. En la socialización realizada por las entidades estatales, y la empresa Cornerstone operadora del denominado proyecto Espejo, una de las comuneras de este sector se refirió al bosque comunitario, puntualizando la importancia de esta área protegida:

"Nosotros somos dueños de nuestra comunidad de La Esperanza, y nosotros rechazaremos a las minas. No lo vamos a permitir, porque el agua viene de nuestro bosque comunal [Cerro Colorado] para algunas comunidades" (pobladora de La Esperanza 1, Socialización Proyecto Minero Espejo, El Chical 9 de agosto del 2017, grabación realizada por autora).

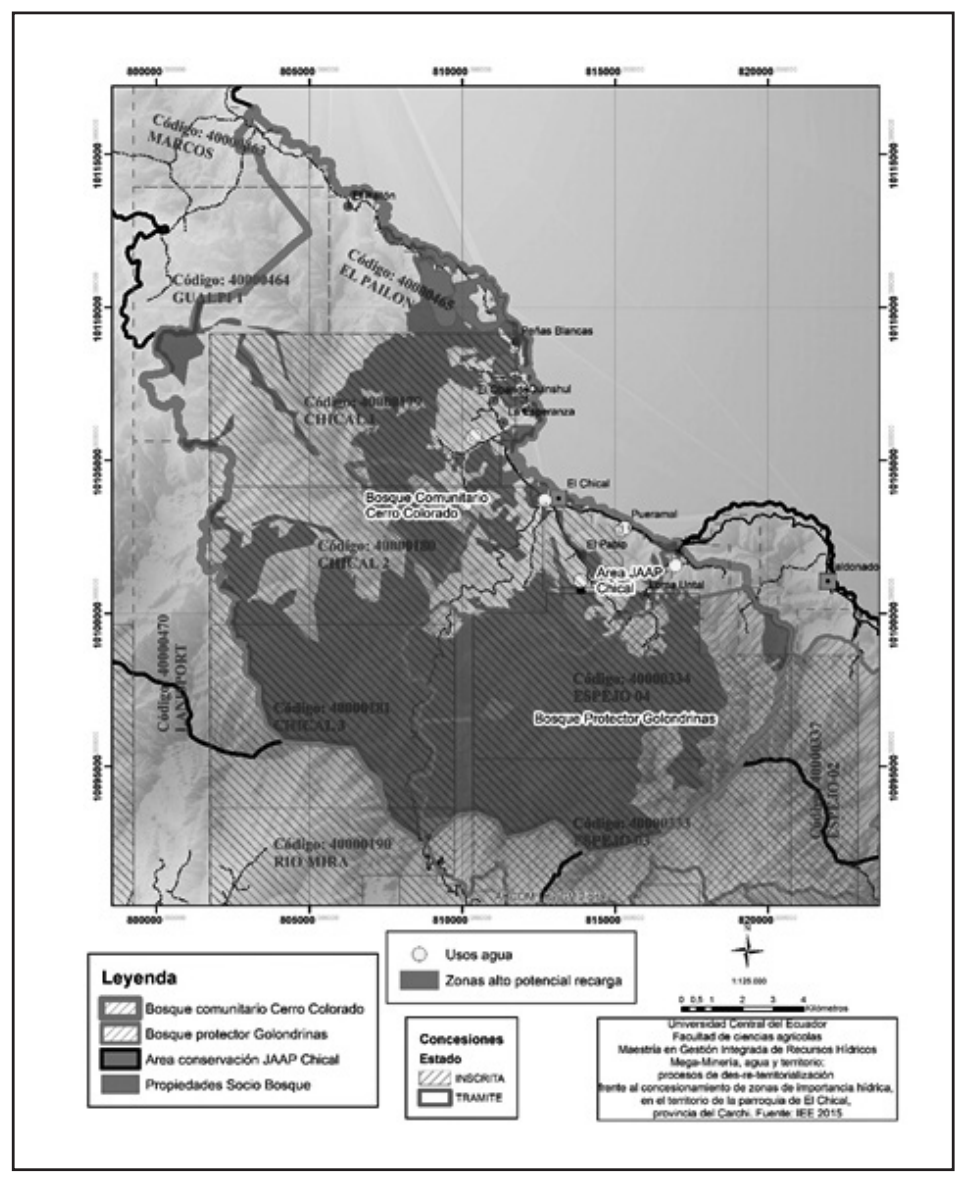

Figura 2. Concesiones minerías, usos del agua y ZHI parroquia El Chical. Elaboración propia a partir de Información 125000 IEE, cantón Tulcán, datos levantados en campo.

En cuanto se realiza un cruce de las zonas de importancia hídrica con las concesiones para megaminería, como se puede observar en la Figura 2 , se constata el claro conflicto por los usos del territorio. Este es el resultado de dos territorialidades en una clara disputa. Además, permite verificar como al momento de realizar la determinación de las áreas posibles para explotación minera no existen consideraciones sobre los posibles alcances de las mismas en relación a la afectación de recursos hídricos.
Las zonas de importancia hídrica propuestas como una categoría de análisis para esta investigación, permitieron identificar áreas trascendentes para mantener la recarga de los sistemas hídricos $\mathrm{y}$, por lo tanto, para abastecer distintos usos y formas de gestión del agua, y sostener las zonas bajo conservación. La identificación de las zonas importantes en términos del agua, con la ayuda de los sistemas de información geográfica, permiten generar representaciones del territorio que se pueden contrastar con las concesiones mineras entregadas. 
Se tiene entonces dos consideraciones en relación al área de estudio. Por un lado, un área que desde una perspectiva de conservación de los recursos hídricos y de los ecosistemas presenta un remanente de bosque nativo altamente amenazado; pero que, además, tiene áreas bajo categorías de conservación reconocidas por el Estado y por las poblaciones locales. Por otro lado, el área es también un territorio habitado por personas, para quienes el agua y los recursos naturales representan la posibilidad de mantener sus modos de vida, que se perciben como en riesgo de ser alterados de manera permanente por la actividad minera a gran escala.

"Nuestra tierra es para vivir, no para [que] el señor presidente nos venga a hacernos daño. Entonces y por esta razón, nuestra tierra, nuestros abuelos y nuestros antepasados nos dejaron para que vivamos y nos mantengamos de la tierra. El oro para nosotros no es vida, el oro es solamente para los gringos, la riqueza que ellos se llevan a otros lados, aqui la gente quedará pobre otra vez. (...) somos oriundos de nuestra tierra; y ustedes no nos van a reponer otro lugar, otro país donde irnos. (...) entonces en vano se han antojado de esta tierra. Este terreno nos lo dejó Dios, y nuestros abuelos, no el presidente de la República" (pobladora de El Chical 8, Socialización Proyecto Espejo, El Chical 9 agosto 2017, grabación realizada por autora).

\section{Conclusiones}

La presente investigación ha buscado analizar los procesos de des-re-territorialización en la zona mestiza de El Chical, a partir de tres grandes temáticas y su interrelación con la problemática agua, minería y territorio. Primero, se delimitó y contextualizó las zonas de importancia hídrica en el área de estudio; luego se analizó las territorialidades locales, el papel del agua en las mismas y las respuestas desde la población local a la imposición de estos territorios mineros.

La megaminería es una actividad que genera cambios irreversibles en los territorios donde se emplaza. En esta investigación se ha profundizado en las afectaciones del agua, en su calidad y cantidad; pero también en las relaciones de la población local con este elemento y cómo éstas se modifican a partir del establecimiento de esta actividad extractiva. El hecho de que zonas de importancia hídrica se encuentren traslapadas con concesiones mineras genera conflictos debido a cómo se está pensando y organizando un mismo territorio desde diferentes intereses. Así, por un lado, el Estado y las empresas mineras construyen una estrategia para legitimar y materializar su presencia en un determinado territorio de su interés; mientras la población también encuentra formas de expresar su descontento y legitimar su territorialidad.

Las zonas de importancia hídrica propuestas como una categoría de análisis para esta investigación, permitieron identificar áreas importantes para mantener la recarga de los sistemas hídricos y, por lo tanto, para abastecer distintos usos y formas de gestión del agua, y sostener las zonas bajo conservación. La identificación de las zonas importantes, en términos del agua, con la ayuda de los sistemas de información geográfica, permiten generar representaciones del territorio que se pueden contrastar con las concesiones mineras entregadas.

A través de la delimitación de las áreas de importancia hídrica se buscó representar de forma espacial, con el uso de cartografía, cómo se distribuyen estas áreas en la zona de estudio. Esto permitió entender de mejor manera cómo se imprimen las territorialidades locales en relación con el agua. Sin embargo, se puntualizó que en esta delimitación cartográfica no fue posible representar algunas aproximaciones locales al agua, como el uso no registrado de fuentes para diversas actividades domésticas, piscícolas, de recreación; o el sentido de pertenencia local relacionado al disfrute y contemplación de los cuerpos hídricos, como parte de las formas de vida locales. Se manifiesta la existencia de una forma local de relacionarse con el elemento agua, tanto a través de las actividades productivas como la piscicultura, de los usos no registrados como la recreación, uso directo del agua de las quebradas para la vida diaria, contemplación y disfrute del agua como algo abundante en su territorio con el que se convive a diario. El agua y los ecosistemas boscosos generan, además, sentido de pertenencia al territorio específico; allí las relaciones sociales y con el entorno, cobran sentido.

Las áreas con alto potencial para recarga fueron identificadas con base en los parámetros desarrollados por Matus, et al. (2009), proceso que también permitió generar algunos criterios de análisis. El primero relacionado al peso que tiene la cobertura vegetal dentro de la fórmula de cálculo aplicada, lo que les confiere a las zonas remanentes de bosque nativo una característica de mayor importancia directamente relacionada a la conservación de los recursos hídricos. Pero, además, al realizar el cruce de estas áreas con las 
zonas bajo categorías de protección, se corrobora que un importante porcentaje de las áreas de alto potencial de recarga se encuentran tanto dentro del Bosque Protector Cerro Golondrinas, como del Bosque Protector Comunitario Cerro Colorado. Esto permite pensar que de alguna forma han existido esfuerzos, tanto desde la población local como desde el gobierno, para establecer zonas de conservación en las cabeceras de cuencas y zonas con remanente de bosque nativo. Sin embargo, se revela también la contradicción entre estas categorías de conservación y las actividades productivas locales, siendo estas áreas indispensables tanto para la manutención de los modos de vida locales, como del equilibrio ecosistémico. Y es que los bosques de vegetación protectores, categoría dentro de la que se encuentra el cerro Golondrinas, indican prohibiciones específicas a las actividades de tala, caza y deforestación, que son practicadas por las poblaciones ubicadas en las áreas de influencia de estas zonas. La contradicción descrita se agudiza si se piensa que el Estado autoriza, en la misma zona y de forma legal, concesiones para megaminería a compañías nacionales o extranjeras, sin mayores requisitos ni contratiempos.

De esta forma se pone sobre la mesa la imposibilidad de ofrecer un nuevo territorio que pueda reemplazar a aquel en donde la población ha ido construyendo sus modos de vida, porque no solamente es valorado desde su capacidad productiva, o la existencia de recursos naturales explotables; el territorio tiene historia y está vivo. Para quienes se reconocen parte del territorio,

\section{Referencias}

ARCOM, Agencia de Regulación y Control Minero. (2018). Catastro Minero. Obtenido de: http:// geo.controlminero.gob.ec:1026/geo_visor/

Bebbington, A., \& Williams, M. (2008). Water and Mining Conflicts in Peru. Mountain Research and Development, 28(3/4), 190-195. https:// doi.org/10.1659/mrd.1039

Budds, J. (2012). Restructuring and Rescaling Water Governance in Mining Contexts: The Co-Production of Waterscapes in Peru, Water Alternatives, 5(1), 119-137.

Burritt, R., \& Christ, K. (2018). Water risk in mining: Analysis of the Samarco dam éste es la evidencia viva del paso de las generaciones, de su trabajo, historia, es su lugar en el mundo.

El proceso de re-territorialización desde los actores locales ha estado marcado inicialmente por el rechazo y descontento expresado en varias de las intervenciones y entrevistas, a pesar del desconocimiento de los procesos de concesionamiento y del avance de los proyectos mineros. Este rechazo al enfrentarse a las estrategias discursivas y económicas, ha generado divisiones en las opiniones y posturas locales. Ello complejiza el plantear la existencia de procesos de re-territorialización claramente marcados, justamente por la agresividad con la que la actividad minera se ha presentado, como la opción de desarrollo para la zona.

Para finalizar, se considera importante presentar a modo de discusión la necesidad de plantear las complejas perspectivas socio económicas en zonas rurales: acceso a educación superior, empleo, trabajo agrícola, que son deudas históricas de la inversión estatal. Hoy en día los conflictos socio ambientales resultado de las políticas extractivistas gubernamentales, no pueden ser solucionados con un abordaje de gobernanza. Es decir, no necesariamente la modificación de políticas relacionadas a la inversión de los excedentes de estas industrias, o al incremento en la trasparencia con la que se manejan estos fondos es lo que la población exige. Para entender esto se debe reconocer las territorialidades diversas, y cómo los grupos territorializados ven en estas actividades extractivas una amenaza de ser desposeídos, y marginados.

failure. Journal of Cleaner Production, 178, 196-205. https://doi.org/10.1016/j. jclepro.2018.01.042

Casellas, A. (2010). La geografía crítica y el discurso de la sostenibilidad. Perspectivas y acciones. Documents d'anàlisi geogràfica, 56(3), 573-581.

Colectivo de Geografia Crítica Ecuador. (2017). Geografiando para la resistencia. Journal of Latin American Geography, 16(1), 172177. Project MUSE. https://doi.org/10.1353/ lag.2017.0006

Fernandes, B.M. (2008). Acerca de la tipología de los territorios (1973), 1-22. 
GAD Parroquial El Chical. (2015). Plan de desarrollo $y$ ordenamiento territorial El Chical. El Chical.

Gobierno Provincial del Carchi. (2015). Plan de Manejo del Bosque Protector Cerro Golondrinas 2015-2020. Tulcán.

Haesbaert, R. (2007). Território e multiterritorialidade: um debate. GEOgraphia - Revista do Programa de Pós-Graduação em Geografia da Universidade Federal Fluminense, 9(17), 1946. https://doi.org/10.22409/GEOgraphia2007. v9i17.a13531

Haesbaert, R. (2013). Del mito de la desterritorialización a la multiterritorialidad. Cultura y Representaciones Sociales, 8(15), 9-42.

Holifield, R., \& Day, M. (2017). A framework for a critical physical geography of 'sacrifice zones': Physical landscapes and discursive spaces of frac sand mining in western Wisconsin. Geoforum, 85, 269-279. https://doi.org/10.1016/j. geoforum.2017.08.004

Matus, O., Faustino, J., \& Jiménes, F. (2009). Guía para la identificación participativa de zonas con potencial de recarga hídrica. Aplicación práctica en la subcuenca del río Jucuapa, Nicaragua. Costa Rica: CATIE (Centro Agronómico Tropical de Investigación y Enseñanza). División de Investigación y Desarrollo (Vol. Serie Técn.).

Ministerio de Minería del Ecuador. (2016). Plan nacional de desarrollo del sector minero. Obtenido de http://www.mineria.gob.ec/plan-nacional-de-desarrollo-del-sector-minero/

Ministerio del Ambiente Ecuador. (2017). Proyecto Socio Bosque. Obtenido de: http://www.ambiente.gob.ec/programa-socio-bosque/

Murillo, D., \& Sacher, W. (2017). Nuevas territorialidades frente a la megaminería: el caso de la Reserva Comunitaria de Junín. Letras Verdes, 22, 46-70. http://dx.doi.org/10.17141/letrasverdes.22.2017.2727

Porto-Gonçalves, C. W. (2009). De Saberes y de Territorios: diversidad y emancipación a partir de la experiencia latino-americana. Polis, Revista de la Universidad Bolivariana, 8(22), 121-136.https://doi.org/10.4067/S071865682009000100008
Raffestin, C. (1980). Por Uma Geografia Do Poder. Sao Paulo: Editora Ática S.A.

Sairinen, R., Tiainen, H., \& Mononen, T. (2017). Talvivaara mine and water pollution: An analysis of mining conflict in Finland. The Extractive Industries and Society, 4(3), 640-651. https:// doi.org/10.1016/j.exis.2017.05.001

Sacher, W. (2017). Ofensiva megaminera en los Andes: acumulación por desposesión en el Ecuador de la "Revolución Ciudadana". Quito: Ediciones Abya-Yala.

Sacher, W., \& Báez, M. (2011). Revisión crítica parcial del "Estudio de Impacto Ambiental para la fase de beneficio del proyecto minero de cobre mirador" de la empresa Ecuacorriente, Ecuador. Quito: Acción Ecológica.

Sacher, W., \& Acosta, Al. (2012). La minería a gran escala en Ecuador. Análisis y datos estadísticos sobre la minería industrial en el Ecuador. Quito: Ediciones Abya-Yala.

Sandoval, L. M. F., Robertsdotter, A., \& Paredes, M. (2017). Space, power, and locality: The contemporary use of territorio in Latin American Geography. Journal of Latin American Geography, 16(1), 43-67. https://doi.org/10.1353/ lag.2017.0009

Silveira, M. M. M., Moreano, M., Romero, N., Muri1lo, D., Ruales, G., \& Torres, N. (2017). Geografías de sacrificio y geografías de esperanza: tensiones territoriales en el Ecuador plurinacional. Journal of Latin American Geography, 16(1), 69-92. https://doi.org/10.1353/lag.2017.0016

Sosa, M., \& Zwarteveen, M. (2012). Exploring the politics of water grabbing: The case of large mining operations in the Peruvian Andes. $\mathrm{Wa}$ ter Alternatives, 5(2), 360-375.

Sosa, M; \& Zwarteveen, M. (2014). The institutional regulation of the sustainability of water resources within mining contexts: Accountability and plurality. Current Opinion in Environmental Sustainability, 11, 19-25. https://doi. org/10.1016/j.cosust.2014.09.013

Vandegrift, R., Thomas, D. C., Roy, B. A., \& Levy, M. (2017). The extent of recent mining concessions in Ecuador. Obtenido de Rainforest In- 
formation Centre: https://ecuadorendangered. $\mathrm{com} / \mathrm{research} /$ reports/RIC-Mapping-Reportv1.1-20180117-eng.pdf.

Yacoub, C. (2007). Identificación y cuantificación de los efectos y consecuencias de los impactos ambientales generados por la mineria de extracción de oro por lixiviación de cianuro.
Caso Mineria Yanacocha SRL, en Cajamarca, Perú. Escola Tècnica Superior d'Enginyeria Industrial de Barcelona - Enginyeria Química.

Yacoub, C. (2013). Developing tools to evaluate the environmental status of Andean basins with mining activities. Tesis Doctoral. Universidad Politecnica de Catalunya. 